\title{
Development of 3D Coupling Analysis Method for Spot Welding by Idealized Explicit FEM*
}

\author{
by Kazuki IKUSHIMA**, Ryohei NATSUME***, and Masakazu SHIBAHARA**
}

\begin{abstract}
In this study, the authors developed a three-dimensional coupling analysis method for spot welding by using Idealized Explicit FEM, which can analyze large-scale welding residual stress and deformation problems in practical computing time. This proposed method was applied to a spot welding problem to show its analysis accuracy. It was found that the nugget sizes obtained by experimental measurements and the method are in good agreement. In addition, to investigate the effect of shunt current on the nugget size in multiple-spot welding, the method was applied to three-dimensional spot welding with four points of welding. As a result, it was shown that the influence of shunt current in multiple-spot welding can be evaluated by using the proposed method in realistic computing time.
\end{abstract}

Key Words: Spot welding, 3D Coupling analysis, Nugget shape, Shunt current, Idealized Explicit FEM

\section{Introduction}

In spot welding, a pair of electrodes makes contact with metal plates and applies electric current to the plates. The Joule heat generated at the contact point heats and welds the metal plates. With spot welding, it is possible to achieve shorter welding time and less thermal strain. In addition, spot welding is easily automated because a skilled worker is not necessary. However, because an empirical approach has traditionally been used to determine welding conditions, the cost and time for experiments are increased. Therefore, using numerical simulations to investigate the welding condition in advance can improve efficiency.

However, the distribution of the current in spot welding changes with the contact state and the heat generation also changes accordingly. Deformation may occur due to heat generation, and the contact state will change accordingly. Therefore, three phenomena of current, heat, and deformation are coupled to each other in spot welding. To analyze spot welding problems by numerical simulations, it is necessary to couple the electrostatic field analysis, the heat conduction analysis, and the thermal-elastic-plastic analysis. These complex coupled analyses require huge memory and computing time.

Due to the difficulty of applying numerical simulations to practical problems, analyses have primarily used a two-dimensional axisymmetric model ${ }^{1)}$. Evaluating the three-dimensional effects of a practical construction model including the shunt current is difficult with a two-dimensional axisymmetric model, because it is impossible to analyze multiple

\footnotetext{
${ }^{*}$ Received: 2014.11.27

*** Member, Graduate School of Engineering, Osaka Prefecture University

${ }^{* * *}$ Student Member, Graduate School of Engineering, Osaka Prefecture University
}

points by axisymmetric model. However, the three-dimensional effect must be considered in practical multiple-spot welding problems because the nugget shapes of subsequent spot welding may change due to the influence of shunt current and deformation by the preceding welding.

Idealized Explicit FEM was developed to analyze large-scale welding residual stress problems ${ }^{2}$. Idealized Explicit FEM is able to analyze three-dimensional welding residual stress in multi-pass welding in realistic computing time, which is very difficult to achieve in the conventional method. In addition, a detailed study of the effects of various factors of welding on the residual stress distribution can be carried out ${ }^{3)}$.

In this study, a three-dimensional simulation method based on Idealized Explicit FEM (IEFEM) is proposed and applied to the spot welding analysis to verify the accuracy of the method. In addition, the method is also applied to a four-point spot welding problem to show the applicability of the method for multiple-spot welding.

\section{Analysis of spot welding by Idealized Explicit FEM}

\subsection{Flow of analysis}

In spot welding, a pair of electrodes makes contact with metal plates. After that, electric current is applied and the Joule heat generated at the contact point heats and welds the metal plates. Figure 1 shows the analyses conducted according to the sequence of spot welding. First, an elastic-plastic analysis is conducted to determine the deformation due to the load on the electrodes. Next, the electrostatic field analysis calculates the electric current field. Subsequently, based on the calculated electric current field, the temperature change due to Joule heat is analyzed by a heat conduction analysis, and then a thermal-elastic-plastic analysis is 


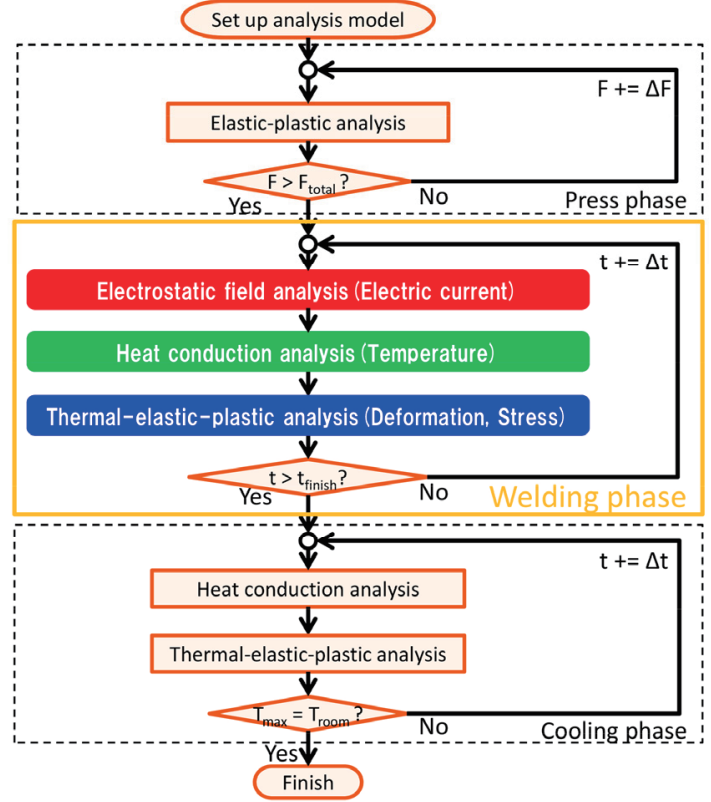

Fig. 1 Analysis flow of spot welding problem.

performed to determine the deformation and stress state due to temperature changes. After heating, the behavior of temperature, deformation and stress on the cooling process is determined by heat conduction analysis and thermal-elastic-plastic analysis. For these two analyses, IEFEM is employed to reduce computing time and memory consumption. The details of the heat conduction analysis and the thermal-elastic-plastic analysis by IEFEM are described in the literature ${ }^{2,4}$. To reduce computing time and memory consumption, IEFEM is also introduced into the electrostatic field analysis, as shown in next section.

\subsection{Electrostatic field analysis}

The governing equations of the electromagnetic field analysis are represented by Maxwell's equations. In the spot welding problem, only the electrostatic field can be considered, because the change of the electromagnetic field over time is small and therefore negligible. In this case, the electrostatic potential $\phi$ can be defined. By using the above assumptions, Maxwell's equations are rearranged according to the following Poisson equation for an electrostatic field:

$$
\varepsilon\left(\frac{\partial^{2} \phi}{\partial x^{2}}+\frac{\partial^{2} \phi}{\partial y^{2}}+\frac{\partial^{2} \phi}{\partial z^{2}}\right)=0
$$

where $\varepsilon$ is the electrical conductivity.

By applying the finite element formulation based on the Galerkin method for Eq. (1), the following finite element equation of the electrostatic field analysis is obtained:

$$
\left[K_{\text {elec }}\right]\{\phi\}=\{q\}
$$

where $\{\phi\},\left[K_{\text {elec }}\right]$, and $\{q\}$ are the nodal electrostatic potential vector, capacitance matrix, and nodal current vector, respectively. These components are derived from the following integrations:

$$
\begin{aligned}
& {\left[K_{\text {elec }}\right]=\int_{\Omega_{i}} \varepsilon\left(\left[\frac{\partial N}{\partial x}\right]^{T}\left[\frac{\partial N}{\partial x}\right]+\right.} \\
& \left.\left[\frac{\partial N}{\partial y}\right]^{T}\left[\frac{\partial N}{\partial y}\right]+\left[\frac{\partial N}{\partial z}\right]^{T}\left[\frac{\partial N}{\partial z}\right]\right) d \Omega \\
& \{q\}=\sum_{i=1}^{N s} \int_{\Gamma_{i}}[N]^{T}[N]\{\hat{q}\} d \Gamma
\end{aligned}
$$

where $[N],[\partial N / \partial x],[\partial N / \partial y],[\partial N / \partial z]$, and $\{\hat{q}\}$ are the shape function of an element, the gradients of the shape function in the $\mathrm{x}, \mathrm{y}, \mathrm{z}$ directions, and the nodal current vector acting on the nodes, respectively. The electric current field is obtained by solving the simultaneous linear equations represented by Eq. (2) under the appropriate boundary condition.

\subsection{Introduction of Idealized Explicit FEM}

The nodal electrostatic potential $\{\phi\}$ can be determined by solving the simultaneous Eq. (2). However, the solution of Eq. (2) may require huge computing time and memory consumption for complex three-dimensional problems. To speed up the computation of these problems, IEFEM is introduced in the electrostatic field analysis.

Analogous to the static elastic analysis, the virtual inertial term and the virtual damping term are added to Eq. (2), which is then modified as follows:

$$
[M]\{\ddot{\phi}\}+[C]\{\dot{\phi}\}+\left[K_{\text {elec }}\right]\{\phi\}=\{q\}
$$

where the first and second terms of the left-hand side are the virtual inertial term and the virtual damping term, respectively, in which $[M]$ and $[C]$ are respectively the virtual mass and the virtual damping matrix. By applying the central difference to Eq. (5), the following equation is obtained:

$$
\begin{gathered}
\left(\frac{1}{\Delta t^{2}}[M]+\frac{1}{2 \Delta t}[C]\right)\{\phi\}_{t+\Delta t}= \\
\{q\}-\left(\left[K_{\text {elec }}\right]+\frac{2}{\Delta t^{2}}[M]\right)\{\phi\}_{t} \\
-\left(\frac{1}{\Delta t^{2}}[M]-\frac{1}{2 \Delta t}[C]\right)\{\phi\}_{t-\Delta t}
\end{gathered}
$$

where $\Delta t,\{\phi\}_{t+\Delta t},\{\phi\}_{t}$, and $\{\phi\}_{t-\Delta t}$ are the virtual time increment and the nodal electrostatic potential vectors at virtual times $t+\Delta t, t$, and $t-\Delta t$, respectively. Here, if the inertial term and the damping term of Eq. (5) are zero, Eq. (5) is 


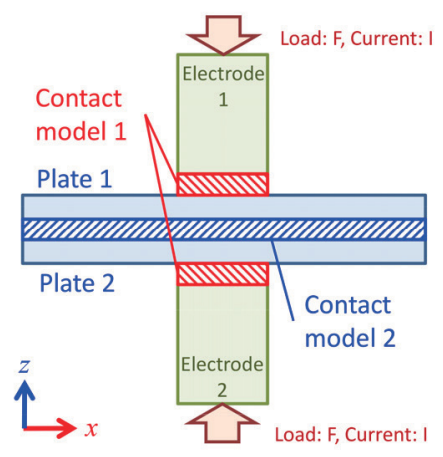

Fig. 2 Schematic illustration of contact model.

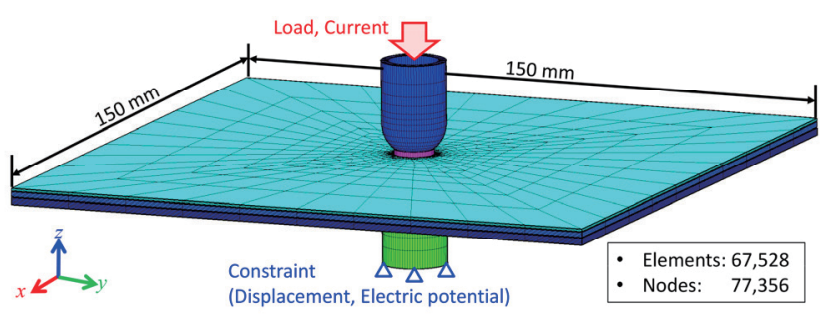

Fig. 3 Analysis model of single-point welding.

equivalent to Eq. (2), which must be satisfied in the electrostatic field analysis. Therefore, it is possible to use Eq. (6) to obtain the solution of Eq. (2) by advancing the time step until the damping term and the inertial term become negligibly small. Mass matrix $[M]$ and damping matrix $[C]$ are determined by considering convergence $^{5)}$. Parallelization by a graphics processing unit (GPU) is also introduced to achieve faster computation, as shown in the literature ${ }^{2)}$.

By using these procedures, computing time and memory consumption of practical three-dimensional problems can be reduced, while the analysis accuracy is almost the same as that of the conventional method.

\subsection{Contact model}

To consider the contact between the plate and the electrode, the contact model shown in Fig. 2 is used. The contact model consists of two types of models: a contact model for the contact between the plate and the electrode, and a contact model for the contact between the two plates. The contact model between the plate and the electrode has large stiffness in the $\mathrm{z}$ direction when the strain on the contact surface is less than -1 , but it has very small stiffness in the $\mathrm{z}$ direction when the strain on the contact surface is larger than -1 . This means that the contact model has a strong reaction force when the electrode and the plate are in contact and no reaction force when the electrode and the plate are separated. When the plates are joined even once, the contact model between the plates has large stiffness in the subsequent analysis.

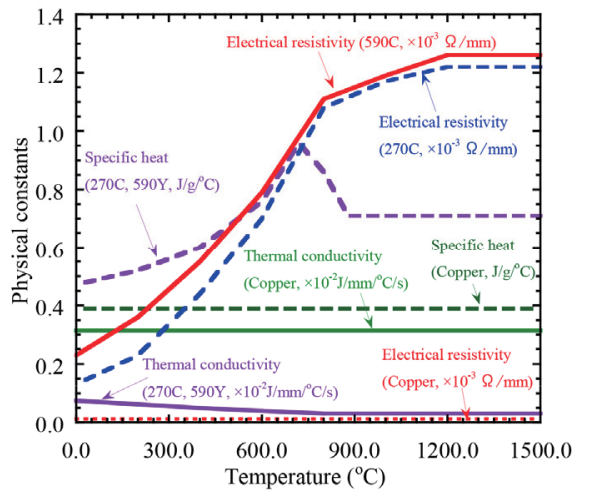

(b) Electrostatic field analysis and heat conduction analysis

Fig. 4 Temperature-dependent material properties.

\section{Verification of proposed method}

\subsection{Analysis model and conditions}

Figure 3 shows the analysis model for verification of the proposed method. The numbers of nodes and elements are 77,356 and 67,528, respectively. The model consists of three plates and copper electrodes. The dimensions of the plates are $150 \mathrm{~mm} \times 150$ $\mathrm{mm}$ and the thicknesses of the upper, middle, and lower plates are $0.7 \mathrm{~mm}, 1.4 \mathrm{~mm}$, and $1.8 \mathrm{~mm}$, respectively. The material of the upper plate is $270 \mathrm{C}$, and that of the middle and the lower plates is 590 Y. Temperature-dependent material constants are shown in Fig. $4^{6}$. Poisson's ratio of $270 \mathrm{C}$ and $590 \mathrm{Y}$ is 0.3 , and that of the copper electrodes is 0.348 . The work hardening coefficient is assumed as 1/200 of the Young's modulus of 270C and 590Y, and no plastic deformation is assumed on the copper electrodes. For the welding conditions, direct current is applied for 17 cycles and each cycle is $20 \mathrm{~ms}$. Three cases are considered for the welding current: $5.0 \mathrm{kA}, 6.0 \mathrm{kA}$, and $7.0 \mathrm{kA}$. The load on the electrodes is set to $2.45 \mathrm{kN}$. The time increment of the heating process is set to $4.0 \times 10^{-4} \mathrm{~s}$. The computer employed for this analysis has Intel Core $173.2 \mathrm{GHz}$ as the CPU, NVIDIA GeForce GTX 580 as the GPU, and 64 GB of memory.

\subsection{Analysis result}

Figures 5 (a), (b), and (c) show the maximum temperature distribution and the nugget for currents $5 \mathrm{kA}, 6 \mathrm{kA}$, and $7 \mathrm{kA}$, respectively. The size of the nugget increases with the electric current. The nugget is defined as the region that has a higher maximum temperature than the melting point of $1530{ }^{\circ} \mathrm{C}$.

Figure 6 shows the relationship between nugget size and current cycle time. The nugget diameter $d$ increases with the current cycle. On the other hand, the nugget thickness $h$ reaches a maximum in the early cycles. It is concluded that the generated heat is absorbed by the electrode immediately, since the 


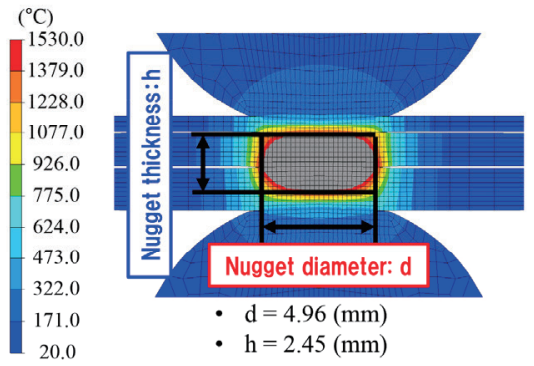

(a) $5 \mathrm{kA}$

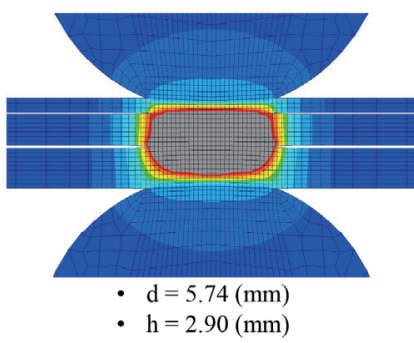

(b) $6 \mathrm{kA}$

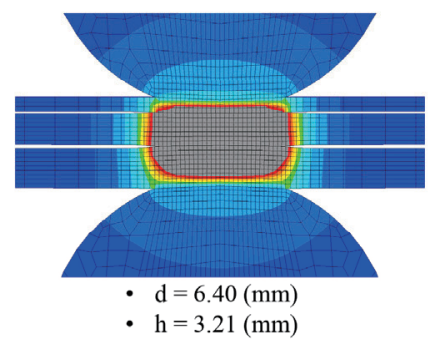

(c) $7 \mathrm{kA}$

Fig. 5 Maximum temperature distribution and shape and size of nugget.

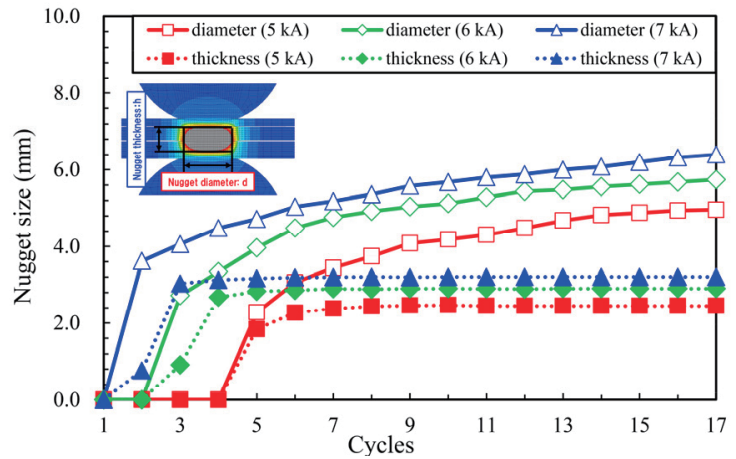

Fig. 6 Relation between current cycle and nugget size for each electric current.

electrodes exist in the thickness direction of the nugget, and the material of the electrodes are copper, which has high thermal conductivity.

Figure 7 compares the relationships between current and nugget size for the experiment ${ }^{6)}$ and the analysis. The nugget sizes of the analysis results and the experimental results are in good agreement.

Because the analysis results agree with the experimental measurements, it can be said that reasonable behavior can be obtained by using the proposed method.

\section{Application to multiple-spot welding}

In multiple-spot welding, it is impossible to use a two-dimensional axisymmetric model because three-dimensional effects such as shunt current are not negligible. For this reason, the proposed method is applied to the problem of four-point spot welding and the applicability of the method to practical three-dimensional problems is shown.

\subsection{Analysis model and conditions}

Figure 8 shows the four-point spot welding model used in this analysis. The numbers of nodes and elements are 98,554 and 85,376 , respectively, and the materials of the electrodes and plates are respectively copper and 590Y. The temperature-dependent material constants are shown in Fig. 4. The dimensions of the plates are $190 \mathrm{~mm} \times 190 \mathrm{~mm}$, and the thicknesses of the upper and

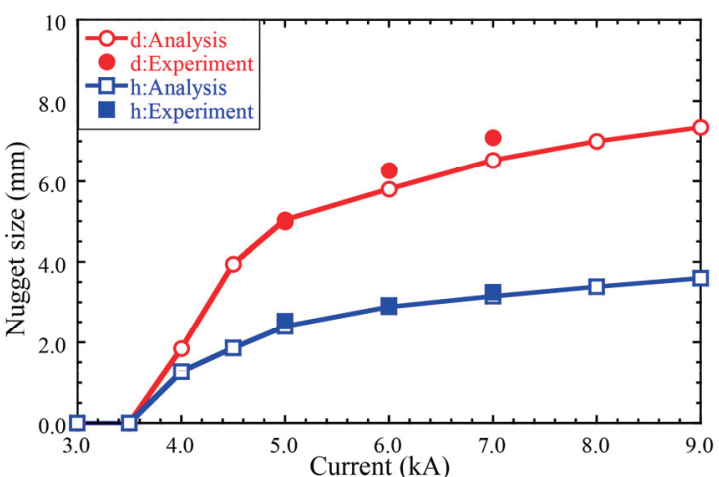

Fig. 7 Relation between electric current and nugget size.

lower plates are $1.4 \mathrm{~mm}$ and $1.8 \mathrm{~mm}$, respectively. The welding current is assumed to be direct current of $7 \mathrm{kA}$, and the load acting on the electrodes is set to $2.45 \mathrm{kN}$. For the heating time, the electric current is applied for 18 cycles and each cycle is 20 $\mathrm{ms}$. The time increment for the heating process is set to $4.0 \times 10^{-4} \mathrm{~s}$ The plates are welded in the order from 1 to 4 as shown in Fig. 8. The computer employed in this analysis is the same as that in the previous section.

\subsection{Analysis result}

Figures 9 (a), (b), (c), and (d) show the maximum temperature distribution for the 1st, 2nd, 3rd, and 4th weld points, respectively. These figures show the nugget shapes. Nugget is defrned as the region that has the maximum temperature reached higher than $1530{ }^{\circ} \mathrm{C}$. The 1 st welding point has the largest nugget of the four points and the nuggets are larger in the order of the 1st, 2nd, 3rd, and 4 th weld points.

Figure 10 compares the nugget sizes for each weld point. The nugget diameter for the 2 nd weld point decreases drastically from that of the 1st weld point and no significant difference is seen between the nugget diameters for the $2 \mathrm{nd}$ and the $3 \mathrm{rd}$ points.

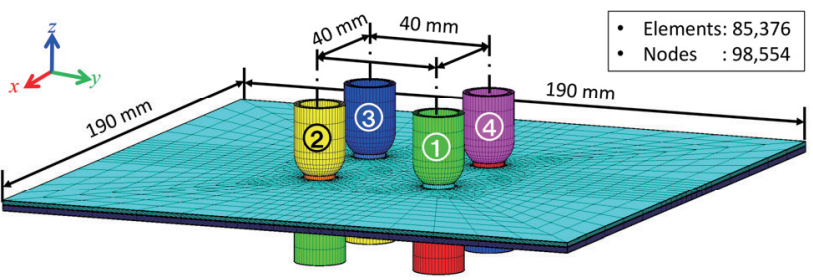

Fig. 8 Analysis model of four-point spot welding. 


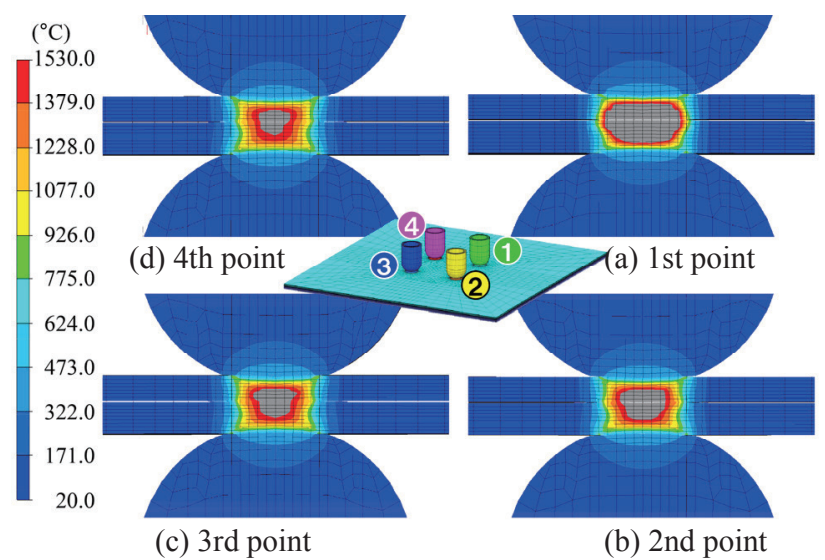

Fig. 9 Maximum temperature distribution on each welding point.

Furthermore, the nugget diameter of the 4th weld point decreases again. The reason for the decrease of the nugget diameter is assumed to be the following: the shunt current does not occur because no weld points are present, and all currents contribute to the nugget formation on the 1st weld point. On the other hand, the shunt current occurs because the 1st weld point has already been welded; therefore, the nugget size becomes smaller on the 2nd weld point. In particular, the Joule heat is proportional to the square of the electric current and a slight change in electric current leads to a large difference in generated heat. Therefore, the nugget diameter is expected to change drastically between the 1 st and 2 nd weld points. In addition, on the 3 rd weld point, the decrease is smaller than that between the 1 st and 2 nd weld points. As shown in Figure 8, the 3rd weld point is separated from the 1st weld point, although the 2nd weld point is close to the 1st weld point. It is therefore assumed that the change of nugget diameter is small, because the influence of shunt current to the 1st weld point is small on the $3 \mathrm{rd}$ weld point. On the 4th weld point, the nugget diameter is reduced because of the increase of shunt current due to the close points of the 1st and 3rd weld points. For the nugget thickness, the same tendency as the nugget diameter can be seen. However, the decrease of nugget thickness is relatively small compared to decrease of diameter. It is assumed that the influence of the shunt current on nugget thickness is smaller than the heat absorption due to the electrodes.

The analysis finished within approximately 10 hours. Therefore, it can be said that the proposed method can examine three-dimensional spot welding problems in practical computing time.

\section{Conclusions}

In this research, the analysis method of spot welding for three-dimensional problems using Idealized Explicit FEM was developed to investigate the influence of shunt current, which may cause problems in spot welding. To verify its validity, the proposed method was applied to the problem of single-point spot

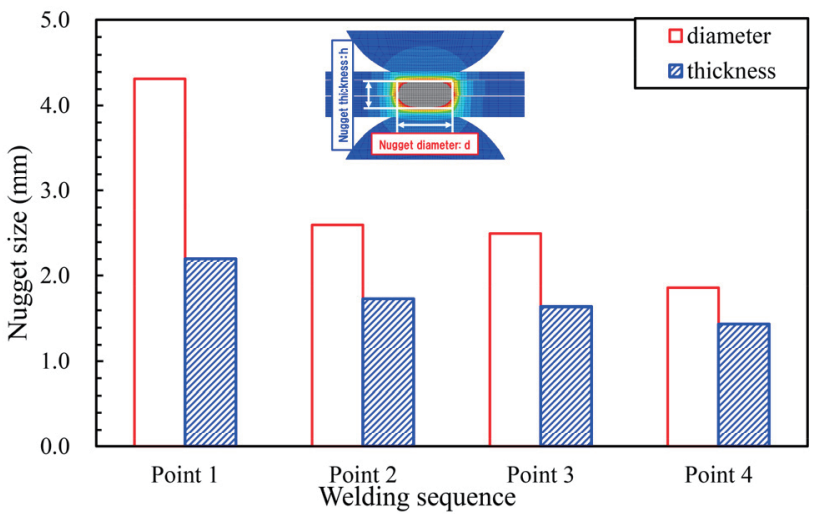

Fig. 10 Comparison of nugget size among all welding points.

welding. Then, to evaluate the effect of shunt current on the formation of the nugget, the method was applied to the analysis of multiple-spot welding. The following results were obtained:

(1) The proposed method was applied to the problem of single-point spot welding and the nugget sizes were compared between experimental measurements and analyzed results. The analysis results agree well with the experimental measurements.

(2) For the analysis of four-point spot welding, the influence of shunt current was investigated by applying the proposed method. As a result, it was found the nugget diameter is reduced after the 2 nd weld point. It was also fonud thae the influence of shunt current on the nugget thickness is smaller than that on the nugget diameter because the heat absorption due to the electrodes is large.

(3) By using the proposed method, the analysis of the three-dimensional problem of spot welding was finished with a practical computing time of 10 hours.

\section{Reference}

1) H. Murakawa, F. Kimura and Y. Ueda: Weldability Analysis of Spot Welding on Aluminum Using FEM, Transactions of JWRI, Vol.24, No.1 (1995), pp.101-111.

2) K. Ikushima and M. Shibahara: Development of Parallelized Idealized Explicit FEM Using GPU, Quarterly Journal of The Japan Welding Society, Vol.31, No.1 (2013), pp.23-32.

3) M. Shibahara, S. Itoh, T. Okada, K. Ikushima and S. Nishikawa: Prediction of Residual Stress in Multi-Pass Welded Joint Using Idealized Explicit FEM, ASME 2012 Pressure Vessels and Piping Conference, No.PVP2012-78357 (2012), pp.667-675.

4) K. Ikushima, S. Itoh and M. Shibahara: Heat Conduction Analysis of Welding Using Idealized Explicit FEM, Quarterly Journal of The Japan Welding Society, Vol.31, No.4 (2013), pp.153-157.

5) K. Ikushima, S. Itoh and M. Shibahara: Numerical Analysis of Welding Deformation for Large-Scale Structure, Quarterly Journal of The Japan Welding Society, Vol.31, No.4 (2013), pp.138-142.

6) N. Ma and H. Murakawa: Numerical and Experimental Study on Nugget Formation in Resistance Spot Welding for Three Pieces of High Strength Steel, Journal of Materials Processing Technology, Vol.210 (2010), pp.2045-2052. 